\title{
A threshold model for local volatility: evidence of leverage and mean-reversion effects on historical data
}

\author{
Antoine Lejay* and Paolo Pigato ${ }^{\dagger}$
}

February 15, 2019

\begin{abstract}
In financial markets, low prices are generally associated with high volatilities and vice-versa, this well known stylized fact usually being referred to as the leverage effect. We propose a local volatility model, given by a stochastic differential equation with piecewise constant coefficients, which accounts for leverage and mean-reversion effects in the dynamics of the prices. This model exhibits a regime switch in the dynamics accordingly to a certain threshold. It can be seen as a continuous-time version of the self-exciting threshold autoregressive (SETAR) model. We propose an estimation procedure for the volatility and drift coefficients as well as for the threshold level. Parameters estimated on the daily prices of 351 stocks of NYSE and S\&P 500, on different time windows, show consistent empirical evidence for leverage effects. Mean-reversion effects are also detected, most markedly in crisis periods.
\end{abstract}

Keywords. Leverage effect; realized volatility; mean-reversion; regime-switch; parametric estimation; threshold diffusion; stock price model

*Université de Lorraine, IECL, UMR 7502, Vandœuvre-lès-Nancy, F-54600, France

CNRS, IECL, UMR 7502, Vandœuvre-lès-Nancy, F-54600, France

Inria, Villers-lès-Nancy, F-54600, France

E-mail: Antoine.Lejay@univ-lorraine.fr

${ }^{\dagger}$ Weierstrass Institute for Applied Analysis and Stochastics, Mohrenstrasse 39, Berlin, 10117, Germany E-mail: paolo.pigato@wias-berlin.de 


\section{Introduction}

Despite the predominance of the Black-Scholes model for the dynamics of asset prices, its deficiencies to reflect all the phenomena observed in the markets are well documented and subject to many studies. Some stylized facts not consistent with the Black-Scholes model are non-normality of log-returns, asymmetry, heavy tails, varying conditional volatilities and volatility clustering (Cont 2001). Regime switching is also consistently observed (Ang \& Timmermann 2012 Salhi et al. 2016). Besides, some assets and indices exhibit mean-reverting effects (see e.g. Meng et al. 2013. Monoyios \& Sarno 2002; Lo \& MacKinlay 1988; Poterba \& Summers 1988; Spierdijk, Bikker \& Hoek 2012).

By considering only the asset's price at discrete times $\{k \Delta t\}_{k=0,1,2, \ldots}$, the logreturns $r_{t}=\log \left(S_{t+1} / S_{t}\right)$ of the Black-Scholes model $\left\{S_{t}\right\}_{t>0}$ are nothing more than the simple time series

$$
r_{t+1}=\left(\mu-\frac{\sigma^{2}}{2}\right) \Delta t+\sigma \sqrt{\Delta t} \epsilon_{t} \text { with } \epsilon_{t} \sim \mathcal{N}(0,1), \text { independent. }
$$

Several models alternative to (1) have been proposed to take some of these stylized facts into account. Among the most popular ones, ARCH and GARCH models and their numerous variants reproduce volatility clustering effects (Engle, Focardi \& Fabozzi 2012).

In this article, we focus on leverage effects, a term which refers to a negative correlation between the prices and the volatility. As observed for a long time, the lower the price, the higher the volatility. First explanations were given in Black (1976) and Christie (1982). Processes such as the constant elasticity volatility (CEV) were proposed to account of these phenomena (Christie 1982). One common economic explanation of leverage effects is that when an asset price decreases, the ratio of the company's debt with respect to the equity value becomes larger, and as a consequence volatility increases; another explanation is that investors tend to become more nervous after a large negative return than after a large positive return; anyway, the origin of leverage effects is still subject to discussion (see e.g. Hens \& Steude 2009).

In the early 1980s, H. Tong has proposed a broad class of time series, the threshold autoregressive models (TAR), with non-linear effects reproducing cyclical data (Tong 1983 Tong 2011; Tong 2015). This class, which contains hidden Markov chains (HMM) as well as self-exciting threshold autoregressive models (SETAR), produces a wide range of behaviors. HMM models rely on a temporal segmentation (they are good for crisis detection), while SETAR models rely on a spatial segmentation, with a regime change when the price goes below or above a threshold.

Time series of SETAR type capture leverage and mean-reverting effects by defining a threshold which separates two regimes (high/low volatility, positive/negative 
trend). Unlike models such as HMM, no external nor latent randomness is used.

In finance, various aspects of SETAR like models have been considered (Yadav, Pope \& Paudyal 1994; Chen, So \& Liu 2011; Meng et al. 2013; Siu 2016; Rabemananjara \& Zakoian 1993). An alternative form to SETAR models is provided by threshold stochastic volatility models (Xu 2012; So, Li \& Lam 2002; Chen, Liu \& So 2008), where the volatility depends non-linearly on the price through a threshold model. Thresholds can also depend on auxiliary variables, as in (Chen \& So 2006). Also other considerations such as psychological barriers (Jang et al. 2015, Kolb 2016) lead to threshold models.

Continuous-time models could be seen as the limit of time series as the time step goes to 0 . They have some advantages over time series, for allowing irregularly sampling, the use of stochastic calculus tools and possibly analytic or semi-analytic formulas for fast evaluation of option prices and risk estimation. Continuous-time threshold models (or threshold diffusion) have been studied in Siu (2016) and $\mathrm{Su}$ \& Chan (2016) for option valuation, in Meng et al. (2013) for portfolio optimization, etc. Self-exciting variants of Vasiček and Cox-Ingersoll-Ross continuous-time models have also been proposed for interest rates (Decamps, Goovaerts \& Schoutens 2006 Pai \& Pedersen 1999). A quasi-maximum likelihood estimator for a threshold diffusion with applications to interest-rate modelling is studied in Su \& Chan (2015) and Su \& Chan (2017). In Brockwell \& Williams (1997), a continuous-time equivalent of an integrated SETAR model is constructed and applied to financial data. Mota \& Esquível (2014) and Esquível \& Mota (2014) propose two continuous-time models which mimic SETAR time series. In Mota \& Esquível (2014), one of these models, referred to as the delay threshold regime switching model (DTRS), is tested on the daily prices of twenty-one companies over almost five years. For almost all the stocks, they find a regime-change for the volatility.

Contribution of the paper. We present the geometric oscillating Brownian motion (GOBM), a threshold local volatility model with piecewise constant volatility and drift, as in Gairat\& Shcherbakov (2016). This model is an instance of the tiled volatility model of Lipton \& Sepp (2011). We stress that the GOBM is the solution of a one-dimensional Stochastic Differential Equation (SDE). Therefore, it is simpler to manipulate than the DTRS of Mota \& Esquível (2014), although having similar features. For the same reason, the market is complete under the GOBM. The GOBM can also be simulated by a standard Euler scheme (Yan 2002 Chan \& Stramer 1998). Option valuation can be performed as well using semi-analytic approaches (Lipton \& Sepp 2011; Lipton 2018: Gairat \& Shcherbakov 2016. Pigato 2017; Decamps, De Schepper \& Goovaerts 2004), and the related problem of estimating ex-ante volatilities from the call prices can be solved using Sturm-Liouville theory (Lipton \& Sepp 2011). 
In the GOBM model, a fixed threshold separates two regimes for the prices. Both the volatility and the drift parameter can assume two possible values, according to the position of the stock price, above or below the threshold. Let us write $\sigma_{-}$for the volatility below the threshold, $\sigma_{+}$for the volatility above the threshold, and similarly $b_{-}$and $b_{+}$for the drift. Such model accounts of the leverage effect, when $\sigma_{-}>\sigma_{+}$. In this case, when prices are low, volatility increases, consistently with what is observed on empirical financial data. As in Mota \& Esquível (2014), the dynamics has two regimes, one corresponding to the bull market, with prices above the threshold and low volatility, and one corresponding to the bear market, with prices below the threshold and high volatility. In this sense, the model displays an "endogenous" regime switch. A motivation for considering such price dynamics coming from a different viewpoint is given in Ankirchner, Blanchet-Scalliet \& Jeanblanc (2017), where it is shown that the GOBM describes the price dynamics corresponding to the optimal strategy for a manager who can control, in a stylized setting, the volatility of the value of a firm, getting bonus payments when the value process performs better than a reference index.

After describing and motivating the model, we consider the estimation of volatilities, drifts and thresholds from discrete observations of historical stock prices. The estimation procedures used in Mota \& Esquível (2014), Esquível \& Mota (2014), and Brockwell \& Williams (1997) are all derived from the ones designed for SETAR time series. Here, we approach the problem directly, proposing an estimation procedure based on stochastic calculus. The estimator of the volatility coefficients is inspired by the integrated volatility/realized variance estimator; for its theoretical analysis we refer the reader to Lejay \& Pigato (2018b). Our estimator can be implemented straightforwardly, differently from the MLE, which is very hard to implement as there is no simple closed form for the density of the GOBM. On the other hand, the estimator of the drift coefficient is the maximum likelihood (MLE) one. Its implementation is also straightforward. Its asymptotic behavior is studied in Lejay \& Pigato (2018a).

In the present paper, we discuss several issues regarding the quality of the estimation and propose a method for estimating the threshold, based on the Akaike information principle. In addition, we provide a hypothesis test to decide whether or not the volatility is constant. We test the performance of such methods via numerical experiments on simulated data. These tests are conclusive.

Finally, we look at empirical financial data. We first benchmark our model against the same dataset as Mota \& Esquível (2014): twenty-one stock prices from the NYSE, on the time window 2005-2009. We find similar results: in particular, we consistently find leverage effects $\left(\sigma_{-}>\sigma_{+}\right)$and mean-reverting behavior $\left(b_{-}>0\right.$ and $\left.b_{+}<0\right)$. Then, we apply our estimators to the empirical time series of the S\&P 500, on the three separate five years windows $2003-2007,2008-2012$ and 
2013 - 2017, finding again consistent evidence of leverage effects. More specifically, we may say, based on the hypothesis test mentioned above and on the estimated ratios $\frac{\sigma_{-}}{\sigma_{+}}$, that the leverage effect is particularly marked in the period $2008-2012$, most likely because it contains the 2008 financial crisis. The mean-reverting behavior is also quite clearly detectable in the 2008 - 2012 period, less so in the periods $2003-2007$ and $2013-2017$, on which $b_{-}$is always positive but $b_{+}$does not display a predominant sign. This seems to be in agreement with the finding that "... the speed at which stocks revert to their fundamental value is higher in periods of high economic uncertainty, caused by major economic and political events", as was shown in Spierdijk, Bikker \& Hoek (2012). We refer to the same paper and to Section 6 for the economic interpretation of this finding.

As final consideration, we remark that the GOBM, despite its extreme simplicity and limited number of parameters, reproduces notable stylized facts of financial markets such as leverage effects and mean-reverting properties. Moreover, the application of the estimators described above to empirical data confirm the presence of such features in the dynamics of financial indices.

Outline. The GOBM is presented in Section 2, In Section 3 we consider the estimation procedures for the volatility (Section 3.1), the drift (Section 3.2) and the threshold (Section 3.3). In Section 4, we benchmark the GOBM model against the DTRS model (in Section 4.1) introduced by Mota \& Esquível (2014) by comparing the estimators on the same data sets (in Section 4.2). In Section 5 , we present a hypothesis test to decide whether a leverage effect is present or not. Finally, in Section 6 we apply our estimators to the stock prices of the S\&P 500, in three consecutive periods of five years, the second one containing the 2008 financial crisis. The article ends with a global conclusion in Section 7.

\section{A threshold model for local volatility}

The model. The geometric oscillating Brownian motion (GOBM) is the solution to the local volatility model

$$
S_{t}=x+\int_{0}^{t} \sigma\left(S_{s}\right) S_{s} \mathrm{~d} B_{s}+\int_{0}^{t} \mu\left(S_{s}\right) S_{s} \mathrm{~d} s,
$$

where $B$ is a Brownian motion and for a threshold $m \in \mathbb{R}$,

$$
\sigma(x)=\left\{\begin{array}{ll}
\sigma_{+} & \text {if } x \geq m, \\
\sigma_{-} & \text {if } x<m
\end{array} \text { and } \mu(x)= \begin{cases}\mu_{+} & \text {if } x \geq m, \\
\mu_{-} & \text {if } x<m .\end{cases}\right.
$$

We use a solution $S$ to (2) as a model for the price of an asset. The log-price 
$X=\log (S)$ satisfies the SDE

$$
X_{t}=x+\int_{0}^{t} \sigma\left(X_{s}\right) \mathrm{d} B_{s}+\int_{0}^{t} b\left(X_{s}\right) \mathrm{d} s
$$

with

$$
\sigma(x)=\left\{\begin{array}{ll}
\sigma_{+} & \text {if } x \geq r, \\
\sigma_{-} & \text {if } x<r
\end{array} \text { and } b(x)= \begin{cases}b_{+}=\mu_{+}-\sigma_{+}^{2} / 2 & \text { if } x \geq r \\
b_{-}=\mu_{-}-\sigma_{-}^{2} / 2 & \text { if } x<r\end{cases}\right.
$$

for a threshold $r=\log (m)$. Notice the slight abuse of notation in (3) and (5), due to the change of the value for the threshold when taking the logarithm.

When the drift $b=0$ and $r=0, X$ is called an oscillating Brownian motion (OBM, Keilson \& Wellner (1978)), a name we keep even in presence of a two-valued drift and a threshold $r \neq 0$. When $\sigma_{+}=\sigma_{-}$and $b_{+}=b_{-}$, the price follows the Black-Scholes model. By extension, we still call the solution to (4) a GOBM.

The effect of the drift is discussed in Section 3.2. When $b_{+}<0$ and $b_{-}>0$, the process is ergodic and mean-reverting. The convergence towards equilibrium differs from the ones in the Vašičeck and Heston models in which the drift is linear.

Existence and uniqueness. The solution to (4) is an instance of a more general class of processes with discontinuous coefficients which was studied in Le Gall (1984). In particular, there exists a unique strong solution to (4), hence to (2).

The (geometric)-OBM can be easily manipulated with the standard tool of stochastic analysis, sometimes relying on the Itô-Tanaka formula instead of the sole Itô formula (see e.g., Étoré 2006).

Properties of the market. Unlike in some regime switching models, there is no hidden randomness leading to incomplete markets, while offering some regime change properties.

Proposition 1. Assuming the GOBM model for the returns process with a constant risk-free rate, the market is viable and complete.

Proof. Using the results of Le Gall (1984), the Girsanov theorem can be applied to the equation for the log-price. Hence, as for the Black-Scholes model, it is possible to reduce the discounted log-price to a martingale by removing the drift. Hence, there exists an equivalent martingale measure, meaning that the market is viable (Jeanblanc, Yor \& Chesney 2009, Theorem 2.1.5.4, p. 89).

As any absolutely continuous measure could only be reached through a Girsanov transform (Le Gall 1984), the risk neutral measure is unique, meaning that the market is complete. 
Remark 1. The affine transform $\Phi(x)=x / \sqrt{\sigma_{-}} \mathbf{1}_{x<m}+x / \sqrt{\sigma_{+}} \mathbf{1}_{x>m}$ transforms the $\log$-price $X_{t}$ into the solution $Y$ to the SDE with local time

$$
Y_{t}=\Phi\left(X_{0}\right)+B_{t}+\int_{0}^{t} \frac{b_{+}}{\sigma_{+}} \mathbf{1}_{x>0}\left(Y_{s}\right) \mathrm{d} s+\int_{0}^{t} \frac{b_{-}}{\sigma_{-}} \mathbf{1}_{x<0}\left(Y_{s}\right) \mathrm{d} s+\kappa L_{t}^{0}(Y)
$$

where $\left(L_{t}^{0}(Y)\right)_{t \geq 0}$ is the local time of $Y$ at position 0 and $\kappa=\left(\sqrt{\sigma_{-}}-\sqrt{\sigma_{+}}\right) /\left(\sqrt{\sigma_{-}}+\right.$ $\left.\sqrt{\sigma_{+}}\right)$. Eq. (6) is a drifted skew Brownian motion (SBM). The local time part cannot be removed by a Girsanov transform (Le Gall 1984). For the SBM, arbitrage may exist as shown in Rossello (2012). The GOBM may be generalized by considering a log-price solution to $\mathrm{d} X_{t}=x+\sigma\left(X_{t}\right) \mathrm{d} B_{t}+b\left(X_{t}\right) \mathrm{d} t+\eta \mathrm{d} L_{t}^{r}(X)$, where $L^{r}(X)$ is the local time of $X$ at the threshold $r$. The effect of the coefficient $\eta \in(-1,1)$ would be to "push upward" (if $\eta>0$ ) or downward (if $\eta<1$ ) the price, which corresponds to some directional predictability effect (Alvarez, Luis \& Salminen 2017). However, considering $\eta \neq 0$ radically changes the structure of the market with respect to classical SDEs.

Monte Carlo simulation. The GOBM at times $k T / n, k=0,1,2, \ldots, n$ is easily simulated by $\bar{S}_{k T / n}=\exp \left(\bar{X}_{k T / n}\right)$ through the recursive equation (Chan \& Stramer 1998; Yan 2002)

$$
\bar{X}_{\frac{(k+1) T}{n}}=\bar{X}_{\frac{k T}{n}}+\sqrt{\frac{T}{n}} \sigma\left(\bar{X}_{\frac{k T}{n}}\right) \eta_{k}+b\left(\bar{X}_{\frac{k T}{n}}\right) \frac{T}{n}, \eta_{k} \sim \mathcal{N}(0,1) \text { independent. }
$$

\section{Estimation of the parameters from the observations of the stock prices}

The GOBM $X$ is defined by five parameters (volatility, drift and threshold, see Table 11) which we are willing to estimate. In Sections 3.1 and 3.2 we consider the estimation of $\left(\sigma_{ \pm}, b_{ \pm}\right)$for fixed threshold $r$, by considering the estimation of the ex-post volatility and of the drift. Afterwards, in Section 3.3 , the threshold is chosen through a model selection principle.

The procedure presented here is simple to implement and provides good results in practice. We stress that the estimators of $\sigma_{ \pm}$can be implemented with no previous knowledge of the drift, and viceversa the estimators of $b_{ \pm}$do not need the knowledge of $\sigma_{ \pm}$to be implemented. The estimators of $\sigma_{ \pm}$are integrated volatility type estimators, simple to implement and widely studied in the framework of SDEs. One could also use a MLE for the volatility, based on discrete observations, but this would require explicit expressions for the transition density, which are very involved for the GOBM. Concerning the estimator for the drift, our MLE (cf. (12)) can be implemented using estimators for local and occupation times proposed in Lejay \& Pigato (2018a), which do not involve the volatility parameter. 


\begin{tabular}{ll}
\hline$S$ & price of the stock \\
$X=\log (S)$ & log-price \\
$\xi=X-r$ & shifted log-price for a threshold $r$ \\
\hline$r$ & threshold of $X$, the log-price \\
$m=\exp (r)$ & threshold of $S$, the price \\
$\sigma_{-}$ & volatility of $X$ below $r$ \\
$\sigma_{+}$ & volatility of $X$ above $r$ \\
$b_{-}$ & drift of $X$ below $r$ \\
$b_{+}$ & drift of $X$ above $r$ \\
$\mu_{-}=b_{-}+\frac{\sigma_{-}^{2}}{2}$ & appreciation rate of $S$ below $m$ \\
$\mu_{+}=b_{+}+\frac{\sigma_{+}^{2}}{2}$ & appreciation rate of $S$ above $m$ \\
\hline$d$ & delay (DTRS only) \\
\hline
\end{tabular}

Table 1: Notations for the GOBM and DTRS models.

\subsection{Estimation of the ex-post volatility}

In this section we consider the estimation of the ex-post volatility for prices given by the model in (2), when the threshold $r=\log (m)$ is known. We recall the estimators and the theoretical convergence results presented in Lejay \& Pigato (2018b), and discuss their application in the framework of volatility modeling. We define $\xi:=X-r=\log (S)-r$ which is a OBM with a threshold at level 0 .

The data. Our observations are $n+1$ daily data $\left\{\xi_{k}\right\}_{k=0, \ldots, n}$ with $\xi_{k}=\log \left(S_{k}\right)-r$ for an a priori known threshold $r$.

Our aim is to estimate $\left(\sigma_{+}, \sigma_{-}\right)$from such observations.

Occupation times. The occupation times below and above the threshold play a central role in our study. The positive and negative occupation times $Q_{T}^{ \pm}$up to time $T$ of $\xi$ as well as their approximations $\mathrm{Q}_{ \pm}(n)$ are

$$
Q_{T}^{ \pm}:=\int_{0}^{T} \mathbf{1}_{ \pm \xi_{s} \geq 0} \mathrm{~d} s \text { and } \mathrm{Q}_{ \pm}(n):=\frac{T}{n} \sum_{i=1}^{n} \mathbf{1}_{ \pm \xi_{i} \geq 0}
$$

The estimators of the volatilities. We write $\xi^{+}:=\max \{\xi, 0\}$ and $\xi^{-}:=$ $-\min \{\xi, 0\}$, the positive and negative parts of $\xi$. Our estimators $\sigma_{ \pm}(n)^{2}$ for $\sigma_{ \pm}^{2}$ are

$$
\sigma_{ \pm}(n)^{2}:=\frac{\left[\xi^{ \pm}, \xi\right]_{n}}{\mathrm{Q}_{ \pm}(n)}
$$


where

$$
\left[\xi^{ \pm}, \xi\right]_{n}:=\sum_{i=1}^{n}\left(\xi_{i}^{ \pm}-\xi_{i-1}^{ \pm}\right)\left(\xi_{i}-\xi_{i-1}\right)
$$

These estimators are natural generalizations of the realized volatility estimators (Barndorff-Nielsen \& Shephard 2002).

Proposition 2 (Lejay \& Pigato 2018b). When $b_{+}=b_{-}=0$, the couple $\left(\sigma_{-}(n)^{2}, \sigma_{+}(n)^{2}\right)$ is a consistent estimator of $\left(\sigma_{-}^{2}, \sigma_{+}^{2}\right)$. Besides, there exists a pair of unit Gaussian random variables $\left(G_{-}, G_{+}\right)$independent of the underlying Brownian motion $B$ (hence of $\xi$ ) such that

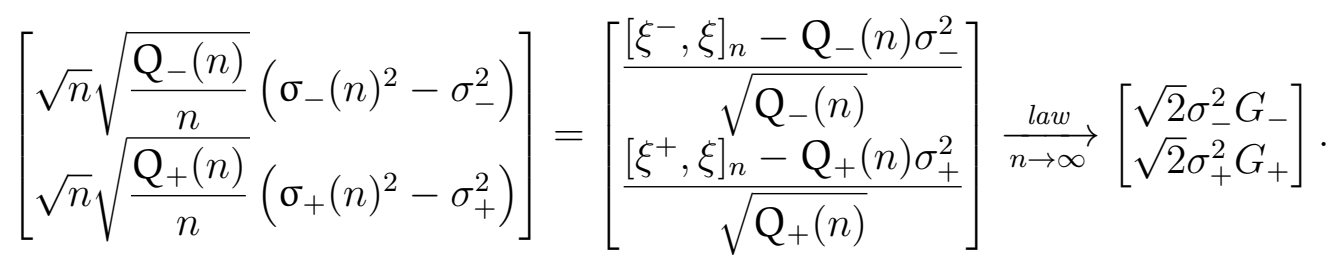

Dealing with a drift. Proposition 2 is actually proved on high-frequency data $\xi_{k, n}:=\xi_{k / n}, k=0, \ldots, n$ on the time interval $[0,1]$.

Using a scaling argument, for any constant $c>0,\left\{c^{-1 / 2} \xi_{c t}\right\}_{t \geq 0}$ is equal in distribution to $\zeta^{(c)}$ solution to the SDE

$$
\mathrm{d} \zeta_{t}^{(c)}=\sigma\left(\zeta_{t}^{(c)}\right) \mathrm{d} W_{t}+\sqrt{c} b\left(\zeta_{t}^{(c)}\right) \mathrm{d} t, \zeta_{0}^{(c)}=c^{-1 / 2} \xi_{0}
$$

for a Brownian motion $W$. With $c=n$, the problem of estimating the coefficients of $\left\{\xi_{k}\right\}_{k=0, \ldots, n}$ is the same as the high frequency estimation of the coefficients of $\left\{\sqrt{n} \zeta_{k, n}^{(n)}\right\}_{k=0, \ldots, n}$ on the time range $[0,1]$.

Without drift, observing $\left\{\xi_{k, n}\right\}_{k=0, \ldots, n}$ or $\left\{\xi_{k}\right\}_{k=0, \ldots, n}$ leads to the same estimation. Using the Girsanov theorem, Proposition 2 stated for the high-frequency regime, that is on the observations $\left\{\zeta_{k, n}^{(1)}\right\}_{k=0, \ldots, n}$ (since all the $\zeta^{(n)}$ are equal in distribution), is also valid in presence of a bounded drift.

With our data, the drift is very small compared to the ex-post volatility and the number $n$ of observations is finite, so that we still apply Proposition 2.

\subsection{Estimation of the drift coefficients}

To estimate the values $b_{ \pm}$of the drift, we consider that the threshold $r=\log m$ is known (this issue is treated in Sect. 3.3. 
Maximum likelihood estimation of the drift. Following Lejay \& Pigato (2018a), we introduce as estimators of $b_{ \pm}$the quantities

$$
\beta_{ \pm}(T):= \pm \frac{\xi_{T}^{ \pm}-\xi_{0}^{ \pm}-L_{T} / 2}{Q_{T}^{ \pm}(\xi)} \text { and } \beta_{ \pm}(n):= \pm \frac{\xi_{T}^{ \pm}-\xi_{0}^{ \pm}-\mathrm{L}(n) / 2}{\mathrm{Q}_{ \pm}(n)}
$$

where $L_{T}$ is the symmetric local time of $\xi$ at 0 while $\mathrm{L}(n)$ is the discrete local time defined by

$$
\mathrm{L}(n):=\sum_{i=0}^{n-1} \mathbf{1}_{\xi_{i} \xi_{i+1}<0}\left|\xi_{i+1}\right| .
$$

The expressions of $\mathrm{L}(n)$ and $\beta_{ \pm}(n)$ do not involve $\sigma_{ \pm}$. Besides, $\beta_{ \pm}(n)$ converges almost surely to $\beta_{ \pm}(T)$ as $n$ goes to infinity.

When the coefficients are constant, as for the log-price in the Black-Scholes model, where $\mathrm{d} \zeta_{t}=\sigma \mathrm{d} B_{t}+b \mathrm{~d} t$, a consistent estimator of the drift $b$ is $b(T)=\left(\zeta_{T}-\zeta_{0}\right) / T$. Our estimator 12 generalizes this formula; the local time term appears because of the discontinuity in the coefficients.

Asymptotic properties. The drift estimator shall be studied for a long time horizon. The asymptotic properties of $\beta_{ \pm}(T)$ as $T \rightarrow \infty$, hence of $\beta_{ \pm}(n)$, depend on the asymptotic behaviors of $Q_{T}^{ \pm}$in $(8)$. We summarize in Table 2 the different cases that depend solely on the respective signs of $b_{+}$and $b_{-}$.

\begin{tabular}{c|ccc} 
& $b_{+}<0$ & $b_{+}=0$ & $b_{+}>0$ \\
\hline$b_{-}>0$ & ergodic (E) & null recurrent (N1) & transient (T0) \\
$b_{-}=0$ & null recurrent (N1) & null recurrent (N0) & transient (T0) \\
$b_{-}<0$ & transient (T0) & transient (T0) & transient (T1) \\
\hline
\end{tabular}

Table 2: Regime of $\xi$ according to the respective signs of $\left(b_{-}, b_{+}\right)$.

The ergodic case, which corresponds to a mean-reverting process, is of course the most favorable one. In the transient case, the estimators may not converge. We briefly recall some of the results in Lejay \& Pigato (2018a) (see the original paper for detailed statements).

$\mathbf{E}$ The ergodic case is the mean-reverting one. In this case $\left(\beta_{-}(T), \beta_{+}(T)\right)$ converges almost surely to $\left(b_{-}, b_{+}\right)$, with speed $\sqrt{T}$.

T0 If $b_{+}>0, b_{-} \geq 0$, then $\beta_{+}(T)$ converges to $b_{+}$with speed $\sqrt{T}$. The estimator $\beta_{-}(T)$ of $b_{-}$does not converge to $b_{-}$and is then meaningless. The case $b_{-}<0$, $b_{+} \leq 0$ is treated by symmetry. 
T1 If $b_{+}>0$ and $b_{-}<0$, then with probability $p:=\sigma_{-} b_{+} /\left(\sigma_{+} b_{-}+\sigma_{-} b_{+}\right), \beta_{+}(T)$ converges to $b_{+}$with speed $\sqrt{T}$, while with probability $1-p, \beta_{-}(T)$ converges to $b_{-}$with speed $\sqrt{T}$. This asymptotic behavior is due to the fact that after a given random time, the process does not cross the threshold anymore.

No Whatever $T>0$ the distribution of $\sqrt{T}\left(\beta_{-}(T), \beta_{+}(T)\right)$ does not depend on $T$. Then $\beta_{ \pm}(T)$ are consistent estimators of $b_{ \pm}=0$.

N1 If $b_{+}=0, b_{-}>0$, then $\left(\beta_{-}(T), \beta_{+}(T)\right)$ converges almost surely to $\left(b_{-}, b_{+}\right)$; $\beta_{-}(T)$ converges to $b_{-}$with speed $T^{1 / 4}$ and $\beta_{+}(T)$ converges to $b_{+}$with speed $\sqrt{T}$.

\subsection{Estimation of the threshold}

The above estimators for $\sigma$ and $b$ assume that the value $m$ of the threshold is known. Following Tong (1983) (see also Priestley 1988, p. 79), we estimate $m$ using a principle of model selection relying on the ideas of the Akaike information principle (AIC) (Akaike 1973). Since the AIC involves the likelihood function, for which we do not necessarily have closed form expressions, we will need to work with approximations.

Approximation of the density. Given a threshold $r$ as well as volatility and drift functions $x \mapsto \sigma(x)$ and $x \mapsto b(x)$, we first consider the density $y \mapsto$ $p(\Delta t, x, y ; r, \sigma, b)$ of $X_{t+\Delta t}$ given $X_{t}=x$ (the process is time-homogeneous so that $p$ only depends on $\Delta t$, not on $t$ ). For a vanishing drift, a closed form expression for $p$ is known (Keilson \& Wellner 1978). In presence of a drift, the expression may become cumbersome if not intractable (Lejay, Lenôtre \& Pichot 2017). However, $p$ can be approximated, in short time, via the related Green function, easier to compute (see Lenôtre 2015, Chapter 2). Alternatively, we assume that the drift is constant over the time interval $[t, t+\Delta t]$ and replace $p$ by the density of $Y_{t+\Delta t}+b(x) \Delta t$ given $Y_{t}=x$, where $Y$ has the same volatility of $X$ yet with a vanishing drift. In the implementation, we use the latter approximation of $p$ which we denote by $\widetilde{p}(t, x, \cdot ; r, \sigma, b)$.

Selection of the threshold. The procedure to select the "best" threshold is then $1 /$ We fix $r^{(1)}, \ldots, r^{(k)}$ possible thresholds in the range of the observed values $\left\{X_{t_{i}}\right\}_{i=0, \ldots, T}$ of the log-price $X$.

2/ For each threshold $r^{(j)}$, we estimate the drift and volatilities $\widehat{\sigma}^{(j)}$ and $\widehat{b}^{(j)}$.

$3 /$ We compute the approximate log-likelihood

$$
\log -\operatorname{Lik}(j)=\sum_{i=0}^{T-1} \log \widetilde{p}\left(t, X_{t_{i}}, X_{t_{i+1}} ; r^{(j)}, \widehat{\sigma}^{(j)}, \widehat{b}^{(j)}\right) .
$$


4/ We select as threshold $\widehat{r}$ the value $r^{(\tilde{\jmath})}$ where $\tilde{\jmath}:=\arg \min _{j=1, \ldots, k} \log -\operatorname{Lik}(j)$.

Comparison with other models. In the model selection based on the AIC, the best model is the one for which the log-likelihood corrected by a value depending on the number of parameters is minimized. Here, the number of parameters is fixed to 4 so that it is sufficient to use only approximations of the log-likelihoods. A similar procedure is used in Meng et al. (2013), yet with a density estimated through Monte Carlo, which is time-consuming. On the contrary, our procedure avoids any simulation step. With respect to the estimation for the SETAR model (Tong 1983 . Priestley 1988), as well as the one of the DTRS model presented below, based on least squares (Mota \& Esquível 2014), there is no delay so that the dimension of the model is reduced by 1 .

\section{Benchmarking the model}

We apply now our estimators to empirical financial data. We benchmark our model against the delay and threshold regime switching model (DTRS) of Mota \& Esquível (2014) by using the same data. We start this section shortly presenting the DTRS model.

\subsection{The delay and threshold regime switching model}

Mota \& Esquível (2014) introduce the DTRS. First, they consider two sets of (functional) parameters $\left(\sigma_{1}, \mu_{1}\right)$ and $\left(\sigma_{2}, \mu_{2}\right)$, as well as a diffusion solution to the stochastic differential equation

$$
\mathrm{d} S_{t}=\mu_{J_{t}}\left(t, S_{t}\right) \mathrm{d} t+\sigma_{J_{t}}\left(t, S_{t}\right) \mathrm{d} B_{t}
$$

for a Brownian motion $B$, where $J$ is a non-anticipative process with values in the set of indices $\{1,2\}$.

The rule for $J$ to switch is based on a threshold $m$, a delay $d$ as well as a small parameter $\epsilon>0$. Assume $S_{0} \leq m$ and $J_{0}=1$. The process evolves according to the parameters $\left(\sigma_{1}, \mu_{1}\right)$ until it reaches the level $m+\epsilon$ at a (random) time $\tau_{1}$. Then it evolves according to the parameters $\left(\sigma_{1}, \mu_{1}\right)$ up to time $\tau_{1}+d$ where it switches to parameters $\left(\sigma_{2}, \mu_{2}\right)$ (that is $\left.J_{\tau_{1}+d}=2\right)$ until it reaches the level $m$ at time $\tau_{2}$. Then it switches again to the state 1 after a delay $d\left(J_{\tau_{2}+d}=1\right)$ and so on.

The parameter $\epsilon$ prevents an accumulation of "immediate" switches so that $S$ can be constructed on a rigorous basis (Esquível \& Mota 2014). With respect to simulation or estimation, $\epsilon$ is of no practical importance as $S$ is only observed or simulated at discrete times. 


\begin{tabular}{rlrl}
\hline AAPL & Apple & ADBE & Adobe \\
AMZN & Amazon & C & CitiGroup \\
CA & CA & CSCO & Cisco \\
GOOG & Google & HP & Hewlett-Packard \\
IBM & IBM & JPM & JP Morgan \\
KO & Coca-cola & MCD & McDonalds \\
MON & Monsanto & MSFT & Microsoft \\
MSI & Motorola & NYT & New-York Times \\
PCG & PG\&E & PFE & Pfizer \\
PG & P \& G & PM & Philip Morris \\
SBUX & Starbucks & & \\
\hline
\end{tabular}

Table 3: Abbreviations of the names of the stocks (in Yahoo Finance).

More specifically, the DTRS model considered in Mota \& Esquível (2014) assumes that the $\mu_{i}$ and $\sigma_{i}(i=1,2)$ are

$$
\left\{\begin{array} { l l } 
{ \sigma _ { 1 } ( t , x ) = \sigma _ { - } \cdot x } & { \text { if } x < m , } \\
{ \sigma _ { 2 } ( t , x ) = \sigma _ { + } \cdot x } & { \text { if } x \geq m }
\end{array} \text { and } \left\{\begin{array}{ll}
\mu_{1}(t, x)=\mu_{-} \cdot x & \text { if } x<m, \\
\mu_{2}(t, x)=\mu_{+} \cdot x & \text { if } x \geq m
\end{array}\right.\right.
$$

for some constants $\sigma_{ \pm}>0$ and $\mu_{ \pm}$. Hence, on each regime, the price $S$ follows a dynamic of Black-Scholes type. We also define $b_{ \pm}=\mu_{ \pm}-\sigma_{ \pm}^{2} / 2$ so that $b_{ \pm}$are the possible values of the drift for the log-price.

Adapting the estimation approach for the SETAR (Tong 1983), Mota \& Esquível (2014) propose a consistent estimation procedure of the parameters, based on least squares.

Results for the DTRS. This estimator is applied to the daily log-prices of twentyone stock prices of the NYSE, from January 2005 to November 2009 (presented in Table 3). In Table 4, we report the estimated values of $\sigma_{ \pm}, m, \mu_{ \pm}\left(\right.$or $\left.b_{ \pm}\right)$and $d$ found in Mota \& Esquível (2014). These values have to be compared with the ones in Table 5 ,

For most of the data, a leverage effect is observed: the ex-post volatility below the threshold is higher than above it. In Mota \& Esquível (2014), option prices of European calls are also computed using a Monte Carlo procedure. The resulting prices are in good agreement with the ones of the market.

Comparison between the DTRS model and the GOBM. In spirit, the GOBM is similar to DTRS of Mota \& Esquível (2014) or to the model in Esquível \& Mota 


\begin{tabular}{|c|c|c|c|c|c|c|c|c|c|}
\hline \multicolumn{10}{|c|}{ Delay threshold regime switching (DTRS) (Mota \& Esquível 2014) } \\
\hline Index & $d$ & $m[\$]$ & $\sigma_{-}[\%] \sigma$ & $+[\%]$ & $\mu_{-}[\%]$ & $\mu_{+}[\%]$ & $b_{-}[\%]$ & $b_{+}[\%]$ & signs \\
\hline AAPL & 8 & 173.5 & 54.1 & 45.6 & 57.5 & -112.4 & 42.8 & -122.7 & +- \\
\hline $\mathrm{ADBE}$ & 1 & 41.5 & 44.0 & 25.1 & 31.8 & -74.3 & 21.9 & -77.4 & +- \\
\hline AMZN & 1 & 77.7 & 51.4 & 45.4 & 54.2 & -462.2 & 41.1 & -472.5 & +- \\
\hline $\mathrm{C}$ & 2 & 43.1 & 120.6 & 16.8 & 39.8 & -3.5 & -32.8 & -5.0 & -- \\
\hline $\mathrm{CA}$ & 1 & 22.1 & 53.0 & 24.6 & 44.4 & -23.2 & 30.2 & -26.2 & +- \\
\hline $\mathrm{CSCO}$ & 1 & 16.3 & 56.0 & 31.3 & 313.5 & 0.0 & 297.6 & -5.0 & +- \\
\hline GOOG & 13 & 642.0 & 37.0 & 40.2 & 40.6 & -148.7 & 33.8 & -156.7 & +- \\
\hline HP & 1 & 46.9 & 39.2 & 27.1 & 42.3 & -78.9 & 34.8 & -82.4 & +- \\
\hline IBM & 1 & 124.3 & 25.1 & 20.3 & 12.9 & -93.5 & 9.6 & -95.5 & +- \\
\hline JPM & 2 & 25.0 & 131.3 & 47.5 & 715.4 & -0.3 & 629.2 & -11.6 & +- \\
\hline $\mathrm{KO}$ & 1 & 10.0 & 78.6 & 29.1 & 398.2 & -5.3 & 367.4 & -9.6 & +- \\
\hline MCD & 1 & 54.6 & 22.4 & 25.7 & 38.8 & -29.5 & 36.3 & -32.8 & +- \\
\hline $\mathrm{MON}$ & 1 & 112.0 & 47.0 & 49.4 & 57.5 & -145.4 & 46.6 & -157.5 & +- \\
\hline MSFT & 1 & 22.9 & 54.0 & 25.1 & 81.1 & 6.0 & 66.5 & 3.0 & ++ \\
\hline MSI & 14 & 21.9 & 49.5 & 28.4 & 7.8 & -47.9 & -4.3 & -51.9 & -- \\
\hline NYT & 4 & 32.5 & 49.5 & 17.3 & -9.8 & -89.5 & -22.2 & -91.0 & -- \\
\hline PCG & 6 & 35.3 & 49.4 & 21.6 & 170.1 & -4.0 & 158.0 & -6.6 & +- \\
\hline PFE & 2 & 16.7 & 40.2 & 24.0 & 67.0 & -14.6 & 59.0 & -17.4 & +- \\
\hline $\mathrm{PG}$ & 1 & 61.9 & 20.3 & 20.2 & 19.2 & -28.0 & 17.1 & -30.0 & +- \\
\hline $\mathrm{PM}$ & 1 & 42.0 & 44.4 & 31.9 & 121.2 & -40.3 & 111.4 & -45.4 & +- \\
\hline SBUX & 15 & 33.6 & 45.4 & 26.0 & 11.6 & -39.8 & 1.3 & -43.1 & +- \\
\hline
\end{tabular}

Table 4: Estimated daily parameters in \% per year found by Mota \& Esquível (2014) for the DTRS model on daily data from January 2005 to November 2009 with the notations given in Table 1 (In the original table, volatilities and drift are expressed in \% per day). The last column signs contains the respective signs of $b_{-}, b_{+}$(a +- indicates a mean-reversion effect).

(2014). Yet, it avoids all the difficulties related to the "gluing" and regime change that involves a very thin layer which serves to avoid infinitely many immediate switches. Hottovy \& Stechmann (2015) discuss the asymptotic behavior of the process as the width of the layer decreases to 0 .

The GOBM has five parameters while the DTRS has six parameters because it also involves a delay. For most of the data, the estimated delay in the DTRS is $d=1$, which means that the switching occurs without delay. Otherwise, the delay means a slow decreasing auto-correlation, or a long memory effect. Yet, for long delay, how to discriminate a leverage effect from sudden changes due to external parameters such as crisis? The presence of a delay increases the possibility of miss-specifications in the estimation procedure. 


\subsection{Estimation of the parameters of the GOBM}

In Table 5, we estimate the parameters for the GOBM on the same stocks as for the DTRS. Although we use the same source (Yahoo Finance) as Mota \& Esquível (2014), it seems that $\mathrm{KO}$ is a different time series than in this article.

The volatilities $\left(\sigma_{-}, \sigma_{+}\right)$are in good agreement for both models. Moreover, $\sigma_{-}>\sigma_{+}$for all the stocks, the only exceptions being MCD for both models and GOOG for the DTRS model. In the latter situation, $\sigma_{-}$is close to $\sigma_{+}$. The respective signs of $b_{-}$and $b_{+}$are consistent with the ones of Mota \& Esquível (2014) and suggest a mean-reversion effect $\left(b_{-}>0, b_{+}<0\right)$ for most of the stock prices. The magnitudes of $b_{-}$and $b_{+}$are also consistent. As the number of data is rather small $(n=1217)$ and the considered period is only five years, it is not reasonable to aim for a more accurate description of the drift. Anyway, this indicates that below the threshold the ex-post volatility is higher and the drift is upward oriented. The threshold estimations are in good agreement for twelve stocks out of twenty-one.

\section{Is there some leverage effect?}

Our aim is to test whether or not $\sigma_{+}=\sigma_{-}$when $b_{-}=b_{-}=0$ (on daily data, $b_{-}$ and $b_{+}$have small values with respect to $\sigma_{-}$and $\sigma_{+}$). Our Hypothesis test is then $\left(H_{0}\right)$ (null hypothesis) $\sigma_{-}=\sigma_{+}$;

$\left(H_{1}\right)$ (alternative hypothesis) $\sigma_{-} \neq \sigma_{+}$.

\subsection{Construction of a confidence region}

The asymptotic result 10 of Proposition 2 is rewritten as

$$
\left[\begin{array}{l}
\sigma_{-}(n)^{2} \\
\sigma_{+}(n)^{2}
\end{array}\right] \approx\left[\begin{array}{l}
\sigma_{-}^{2} \\
\sigma_{+}^{2}
\end{array}\right]-\frac{1}{\sqrt{n}} M_{T} \mathbf{G} \text { with } M_{T}=\sqrt{2 T}\left[\begin{array}{cc}
\sigma_{-}^{2} / \sqrt{Q_{T}^{-}} & 0 \\
0 & \sigma_{+}^{2} / \sqrt{Q_{T}^{+}}
\end{array}\right]
$$

where $\mathbf{G} \sim \mathcal{N}(0$, Id $)$ is a Gaussian vector independent of the observed process $\xi$. The limit term in (16) involves a double randomness, and $M_{T}$ is a measurable function of $\xi$. We approximate $M_{T}$ by

$$
\mathrm{M}(n):=\sqrt{2 T}\left[\begin{array}{cc}
\sigma_{-}^{2}(n) / \sqrt{\mathrm{Q}_{-}(n)} & 0 \\
0 & \sigma_{+}^{2}(n) / \sqrt{\mathrm{Q}_{+}(n)}
\end{array}\right] .
$$

As the Gaussian vector $\mathbf{G}$ is isotropic, we define for a level of confidence $\alpha$ the quantity $q_{\alpha}$ by $\mathbb{P}\left[|\mathbf{G}| \leq q_{\alpha}\right]=1-\alpha$. This quantity is easily computed since $|\mathbf{G}|^{2}$ 


\begin{tabular}{crrrrrrrr}
\hline Index & $m[\$]$ & $\sigma_{-}[\%]$ & $\sigma_{+}[\%]$ & $\mu_{-}[\%]$ & $\mu_{+}[\%]$ & $b_{-}[\%]$ & $b_{+}[\%]$ & signs \\
\hline AAPL & 119.4 & 59.86 & 40.48 & 43.06 & 19.66 & 25.14 & 11.46 & ++ \\
ADBE & 46.1 & 46.79 & 81.37 & 13.93 & -136.87 & 2.98 & -169.97 & +- \\
AMZN & 39.7 & 38.71 & 54.57 & 36.72 & 36.59 & 29.23 & 21.70 & ++ \\
C & 40.1 & 118.58 & 17.35 & -37.30 & -7.61 & -107.60 & -9.11 & -- \\
CA & 21.5 & 51.27 & 25.54 & 40.35 & -10.56 & 27.21 & -13.82 & +- \\
CSCO & 16.9 & 60.48 & 30.70 & 156.08 & 0.05 & 137.79 & -4.67 & +- \\
GOOG & 373.8 & 44.69 & 33.03 & 86.76 & 6.06 & 76.77 & 0.60 & ++ \\
HP & 57.6 & 66.18 & 41.09 & 53.51 & -125.14 & 31.61 & -133.58 & +- \\
IBM & 115.2 & 26.04 & 20.21 & 17.86 & -20.97 & 14.47 & -23.01 & +- \\
JPM & 32.5 & 130.32 & 41.54 & 233.41 & -2.13 & 148.48 & -10.76 & +- \\
KO & 47.8 & 23.70 & 17.88 & 11.75 & 5.72 & 8.94 & 4.12 & ++ \\
MCD & 51.4 & 20.23 & 28.02 & 29.42 & 3.54 & 27.37 & -0.39 & +- \\
MON & 85.8 & 52.71 & 55.80 & 63.67 & -99.35 & 49.78 & -114.92 & +- \\
MSFT & 23.2 & 51.20 & 25.90 & 74.67 & -6.49 & 61.56 & -9.84 & +- \\
MSI & 14.2 & 66.44 & 25.99 & -7.71 & -1.10 & -29.79 & -4.48 & -- \\
NYT & 16.0 & 78.46 & 25.85 & -7.40 & -25.26 & -38.18 & -28.60 & -- \\
PCG & 33.3 & 127.09 & 23.29 & 504.83 & 4.24 & 424.06 & 1.53 & ++ \\
PFE & 19.1 & 39.68 & 20.55 & 11.92 & -7.54 & 4.05 & -9.65 & +- \\
PG & 51.9 & 29.62 & 20.12 & 39.25 & 3.14 & 34.87 & 1.11 & ++ \\
PM & 41.9 & 45.37 & 30.81 & 141.64 & -45.60 & 131.35 & -50.35 & +- \\
SBUX & 13.0 & 71.88 & 46.51 & 49.37 & -13.51 & 23.54 & -24.33 & +- \\
\hline
\end{tabular}

Table 5: Estimated parameters in \% per year for the GOBM model on the daily data from January 2005 to November 2009 with the notations given in Table 1. The last column signs contains the respective signs of $b_{-}, b_{+}$(a +- indicates a mean-reversion effect).

follows a $\chi^{2}$ distribution with two degrees of freedom. Our confidence region of level $\alpha$ is the ellipsis

$$
\mathcal{R}_{\alpha}=\left\{\left[\begin{array}{c}
\sigma_{-}(n)^{2} \\
\sigma_{+}(n)^{2}
\end{array}\right]+\frac{q_{\alpha}}{\sqrt{n}} \mathrm{M}(n)\left[\begin{array}{c}
\cos (\theta) \\
\sin (\theta)
\end{array}\right] \mid \theta \in[0,2 \pi)\right\} .
$$

Our rule of decision is then: reject the Null Hypothesis $\left(H_{0}\right)$ if the diagonal line $s:[0,+\infty) \mapsto(s, s)$ does not cross $\mathcal{R}_{\alpha}$.

\subsection{Numerical simulations}

We perform numerical simulations to check the reliability of the estimation of $\left(\sigma_{-}, \sigma_{+}, r\right)$ as well the hypothesis test " $\sigma_{+}=\sigma_{-}$". For this, we simulate $N=1000$ 


\begin{tabular}{llccccc}
\hline & & $\sigma_{-}$ & $\sigma_{+}$ & $\mu_{-}$ & $\mu_{+}$ & $m$ \\
\hline set 1 & $\sigma_{+} \ll \sigma_{-}$ & $80 \% / \mathrm{yr}$ & $30 \% / \mathrm{yr}$ & 0 & 0 & 1 \\
set 2 & $\sigma_{+} \approx \sigma_{-}$ & $50 \% / \mathrm{yr}$ & $30 \% / \mathrm{yr}$ & 0 & 0 & 1 \\
set 3 & $\sigma_{+}=\sigma_{-}$ & $30 \% / \mathrm{yr}$ & $30 \% / \mathrm{yr}$ & 0 & 0 & 1 \\
\hline \multicolumn{5}{c}{$S_{0}=1 \$$} \\
\hline
\end{tabular}

Table 6: Set of yearly parameters used for simulations.

\begin{tabular}{rcc}
\hline$\sigma_{+} \ll \sigma_{-}$ & $\sigma_{+} \approx \sigma_{-}$ & $\sigma_{+}=\sigma_{-}$ \\
\hline $81 \%$ & $81 \%$ & $14 \%$ \\
\hline
\end{tabular}

Table 7: Proportion of rejection of the null hypothesis $\left(H_{0}\right)$ " $\sigma_{+}=\sigma_{-}$" with a $95 \%$ confidence level for the three sets of parameters of Table 6, with 1000 simulations per set.

paths with daily data over five years for the three sets of parameters given in Table 6. The density of the estimated values of $\sigma_{-}, \sigma_{+}$and $r$ are shown in Figure 1 The proportion of rejection of the hypothesis test " $\sigma_{+}=\sigma_{-}$" are given in Table 7 A good agreement is then observed for the parameters $\sigma_{-}, \sigma_{+}$and the threshold.

The estimation of $\mu_{+}$and $\mu_{-}$, not shown here, presents a large variance, as expected, since when $\mu_{+}=\mu_{-}=0$ the process is only null recurrent. This is discussed in full details in Lejay \& Pigato (2018a).

\subsection{Empirical result on the 2005 - 2009 data}

As the drift is small, it should not affect this test. Therefore we assume through all this section that $b_{-}=b_{+}=0$.

In Figure 2, we apply this rule to our data. The null hypothesis $\left(H_{0}\right)$ " $\sigma_{-}=\sigma_{+}$" is rejected for all the stocks except for PCG, meaning that $\sigma_{-} \neq \sigma_{+}$should be considered for twenty out of twenty-one stocks. The normalized occupation time $O_{+}$ for PCG is close to $99 \%$. This may explain the elongated shape of the associated confidence region.

In Figure 3, we plot the approximated log-likelihood $\log -\operatorname{Lik}(i)$ against $r^{(i)}=$ $\log m^{(i)}$ for three stocks. We see that Log-Lik $(i)$ may have one main peak (for CSCO), two main peaks (for GOOG) or be "flat" as for PCG. A steep peak means that it is clear where the threshold level should be taken, and the procedure is more stable. In these cases, $\sigma_{-}$is likely to differ from $\sigma_{+}$and leverage effect occurs. 

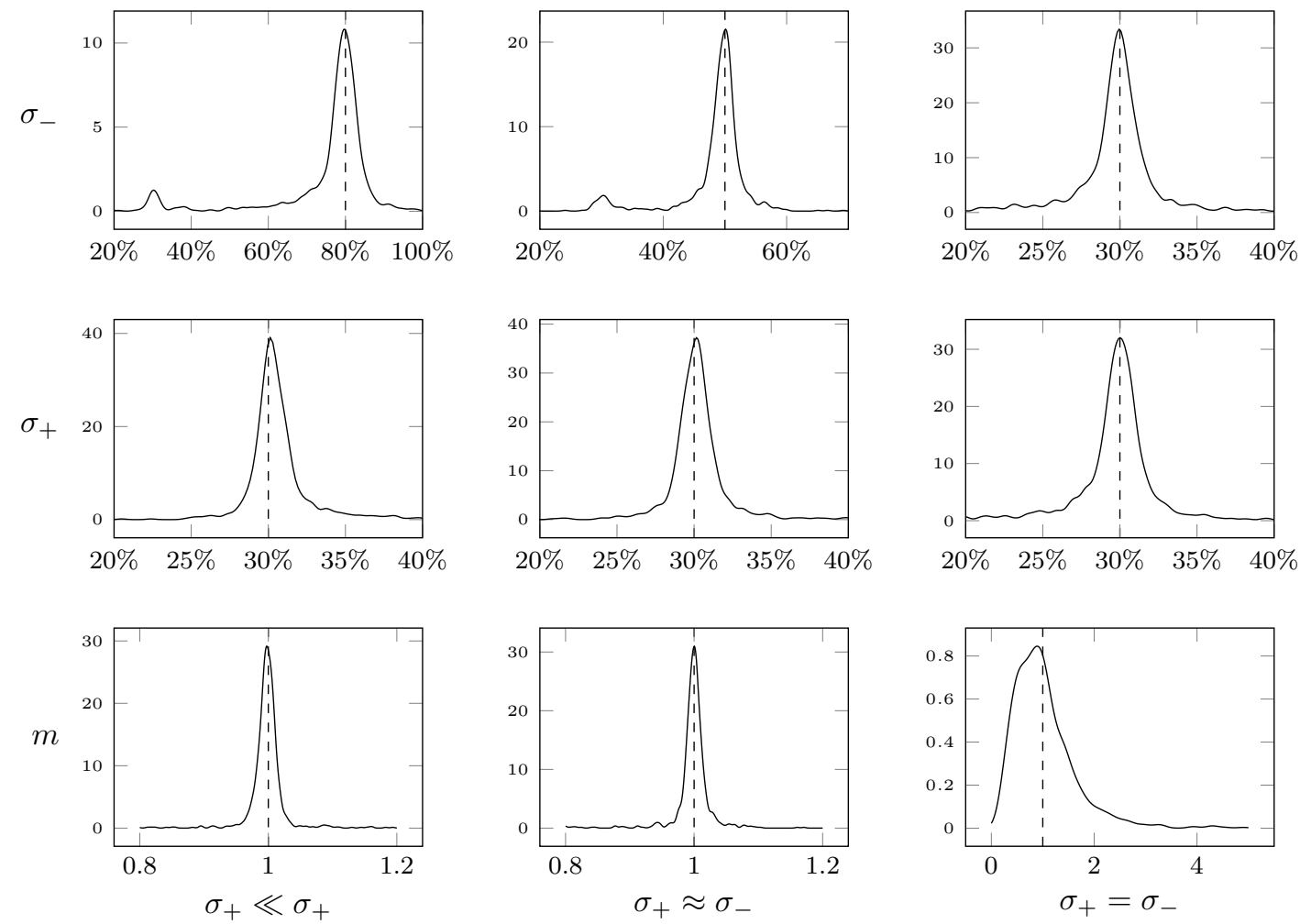

Figure 1: Density of the estimated values of $\sigma_{-}, \sigma_{+}$(yearly) and the threshold $m$ for the three sets of parameters of Table 6, with 1000 simulations per set.

\subsection{Comparison with a non-parametric estimator}

Non-parametric estimation assumes nothing on the underlying volatility and drift coefficients (Kutoyants 2004 Iacus 2008). The Nadaraya-Watson estimator provides us with such an estimator (Iacus 2008). We then compare graphically our estimations with the non-parametric estimation of the coefficients of the log-price. For this, we use the $\mathrm{R}$ package sde (Iacus 2008). In Figure 4, we present the results for the three stocks already used in Figure 3. More figures may be found in Lejay \& Pigato (2017). Most of the stocks seem to exhibit a behavior similar to the one presented here, with a sharp variation of both the volatility and the drift. Again, this reinforces the idea that regime switching holds for most of the stocks. 


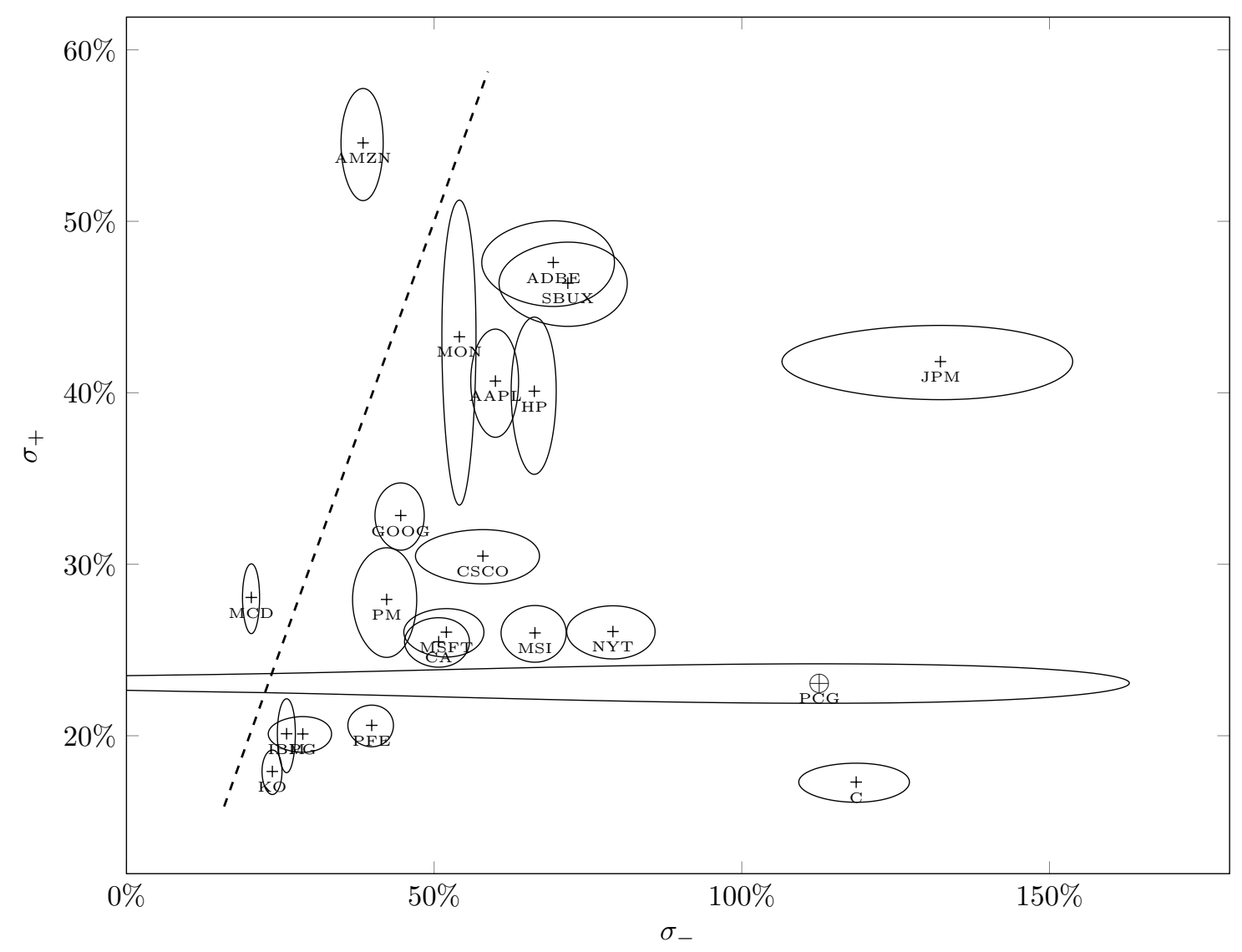

Figure 2: Confidence regions for $\left(\sigma_{-}, \sigma_{+}\right)$: Each point is the value of the stock in the $\left(\sigma_{-}, \sigma_{+}\right)$-plane. Confidence regions at $95 \%$ are the ellipsis in the $\left(\sigma_{-}, \sigma_{+}\right)$-plane around the points. Points marked by $\oplus$ are the ones for which the Hypothesis $\sigma_{-}=\sigma_{+}$is not rejected. Points marked by + are the ones for which this Hypothesis is rejected.

\section{Leverage and mean-reversion effects for the S\&P 500 stocks}

We apply our estimators to the $\mathrm{S} \& \mathrm{P} 500$ stock prices over the three periods of five years each: $1 / 1 / 2003-31 / 12 / 2007,1 / 1 / 2008-31 / 12 / 2012$ and $1 / 1 / 2013-$ $31 / 12 / 2017$. The second period contains the 2008 financial crisis.

Estimators are thus computed on the three periods for 332 of the stocks out of the 500, failures being due to the lack of complete data or to stock prices with at most $5 \%$ of the observations on each side of the estimated threshold. The latter 

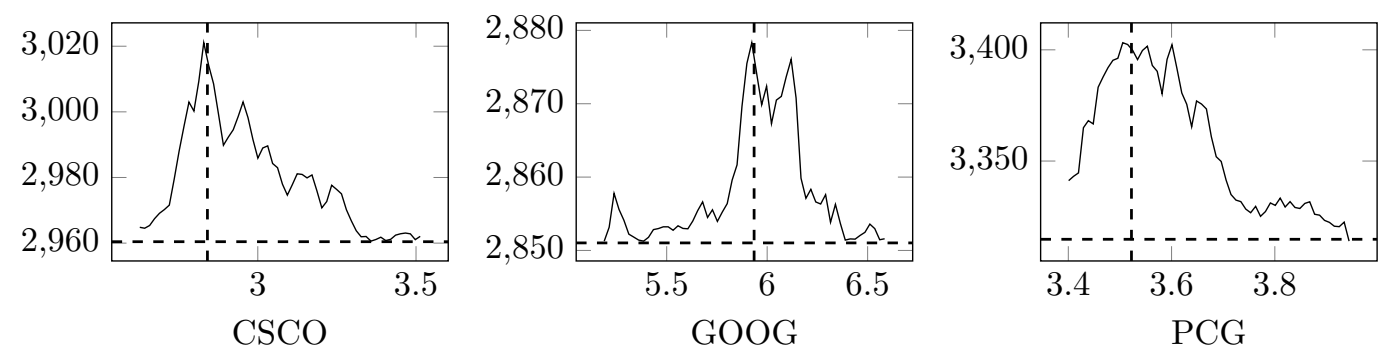

Figure 3: The (approximated) log-likelihood $\log -\operatorname{Lik}(i)$ given by (13) against the possible threshold $r^{(i)}=\log m^{(i)}$ for the stocks CSCO, GOOG and PCG. The vertical dashed line represents the threshold $r^{(i)}$ which maximizes Log-Lik $(i)$, hence the estimated $r$. The horizontal dashed line represents the value of the log-likelihood of the drifted Brownian motion (that is $\sigma_{-}=\sigma_{+}$and $b_{-}=b_{+}$).
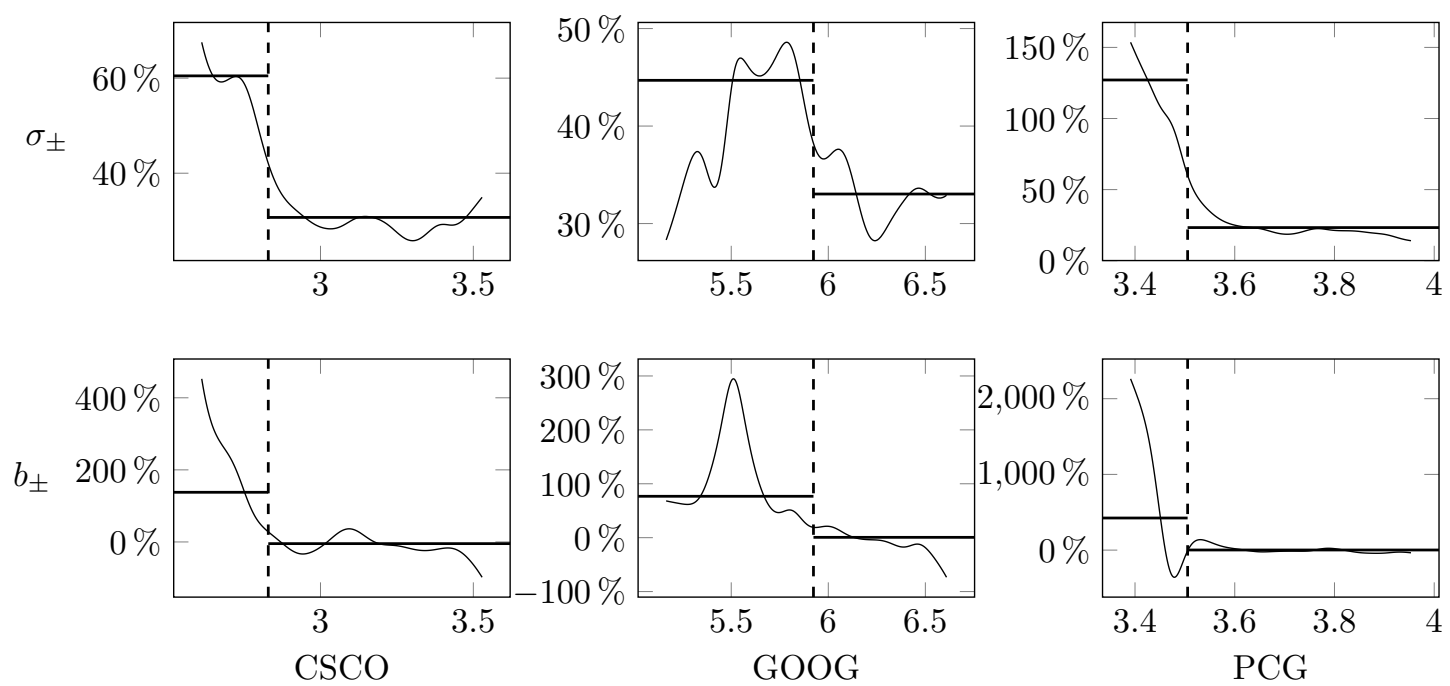

Figure 4: Non-parametric estimation of $\sigma$ and $b$ for the log-price of the stocks CSCO, GOOG and PCG with a Nadaraya-Watson estimator. The vertical dashed line represents the choice of the threshold. The horizontal lines represent the estimated values of $\left(\sigma_{-}, \sigma_{+}\right)$(top) and $\left(b_{-}, b_{+}\right)$(bottom).

exclusion aims at avoiding outliers.

We plot the estimated values of $\left(\sigma_{-}, \sigma_{+}\right)$and $\left(b_{-}, b_{+}\right)$in Figure 5 .

In the 2008-2012 period, containing the 2008 financial crisis, the stock prices exhibit higher ratio of $\sigma_{-} / \sigma_{+}$, hence stronger leverage effects. For the 2008-2012 

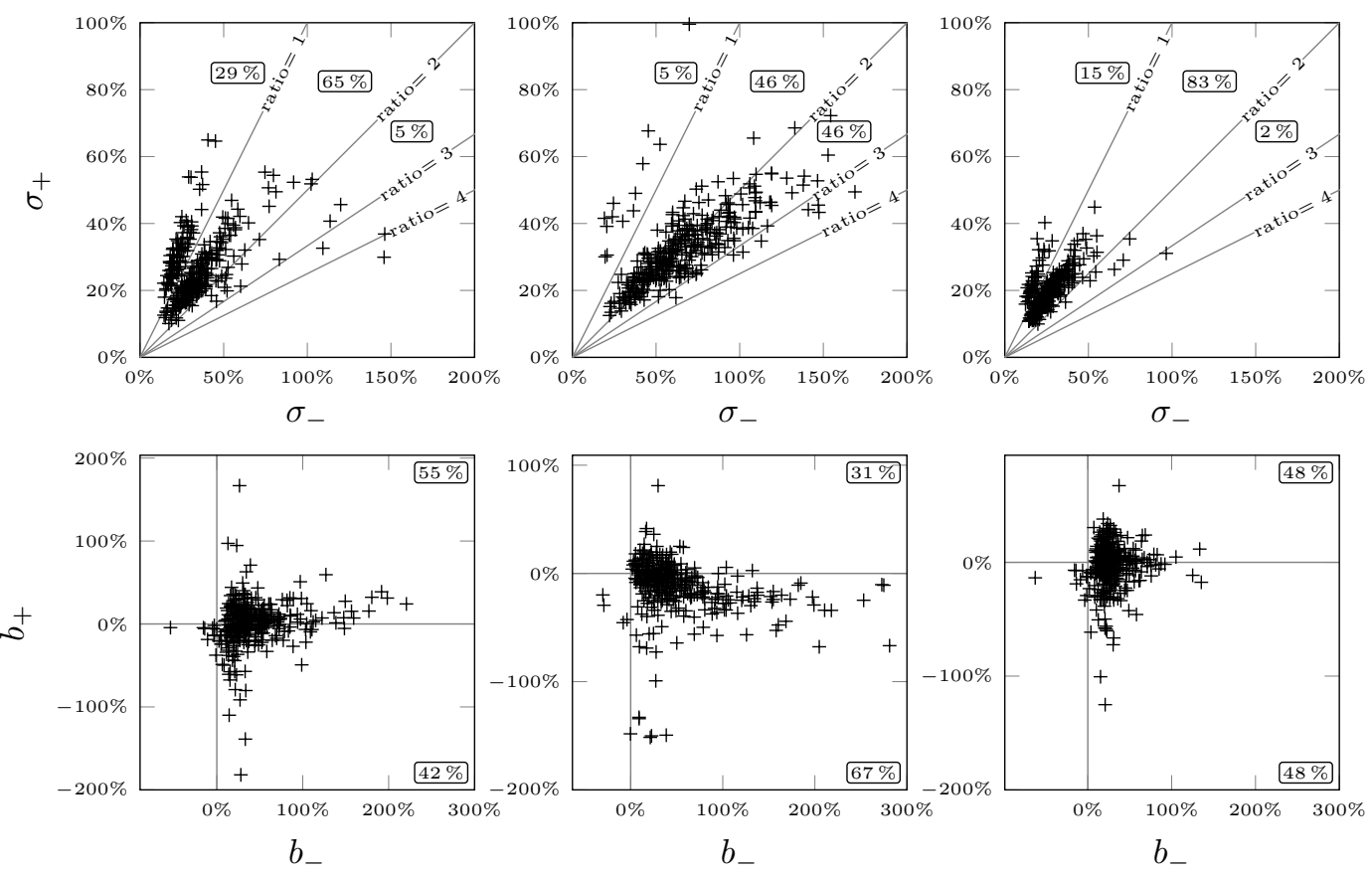

$01 / 01 / 2003-31 / 12 / 2007$

$01 / 01 / 2008-31 / 12 / 2012$

$01 / 01 / 2013-31 / 12 / 2017$

Figure 5: Yearly estimated values of $\left(\sigma_{-}, \sigma_{+}\right)$and $\left(b_{-}, b_{+}\right)$on the daily close prices for the stocks in the S\&P 500. The percentages in boxes indicates the proportions of stocks in each region delimited by the solid gray lines.

period, the hypothesis test " $\sigma_{-}=\sigma_{+}$" is rejected for all the stocks. For the 2003-2007 period (resp. 2013-2017), the test is rejected for all but five (resp. six) stocks.

These findings seem to be consistent with Aït-Sahalia, Fan \& Li (2013), which shows leverage effect in an aggregated form through the correlation between the VIX (involving the ex-ante volatility) and the log-return of the indices of the S\&P 500 for the period $1 / 1 / 2004-12 / 12 / 22007$.

Let us now look at the drift coefficient. First, we notice that $b_{-}$is almost always positive, but $b_{+}$can be positive or negative. For the 2008-2012 period, which contains the 2008 financial crisis, 224 stocks show a mean-reverting behavior $\left(b_{-}>0, b_{+}<0\right)$ against 140 (resp. 161) for the 2003-2007 period (resp. the 20132017). A possible interpretation of such results is that, on periods not involving financial crisis, prices tend to increase in time and therefore also the estimated value of $b_{+}$is positive relatively often. When a crisis occurs, prices oscillate more, going down as well as up, and thus giving in most cases estimated values of $b_{+}<0$, $b_{-}>0$. This seems to be consistent with the results of Section 4 and Mota \& 
Esquível (2014).

Let us also report here a similar finding of Spierdijk, Bikker \& Hoek (2012), together with one of its economic interpretations given in the same paper: "Our findings suggest that expected returns diverge away from their long-term value and converge back to this level relatively quickly during periods of high economic uncertainty; much faster than in more tranquil periods. When the economic uncertainty dissolves, expected returns are likely to show a substantial increase in value during a relatively short time period, which could account for such high mean-reversion speed. Measures and interventions by financial and government institutions to restore financial stability may also speed up the adjustment process."

In Figure 6, we plot a normalized log-threshold by dividing the log-threshold $r$ by the mean log-price (in order to get a normalized value) for one period against the next one. We observe that this ratio ranges between 0.8 and 1.2 for each stock and each period. The normalized log-threshold is statistically higher for the 2013-2017 period than for the 2008-2012 period.
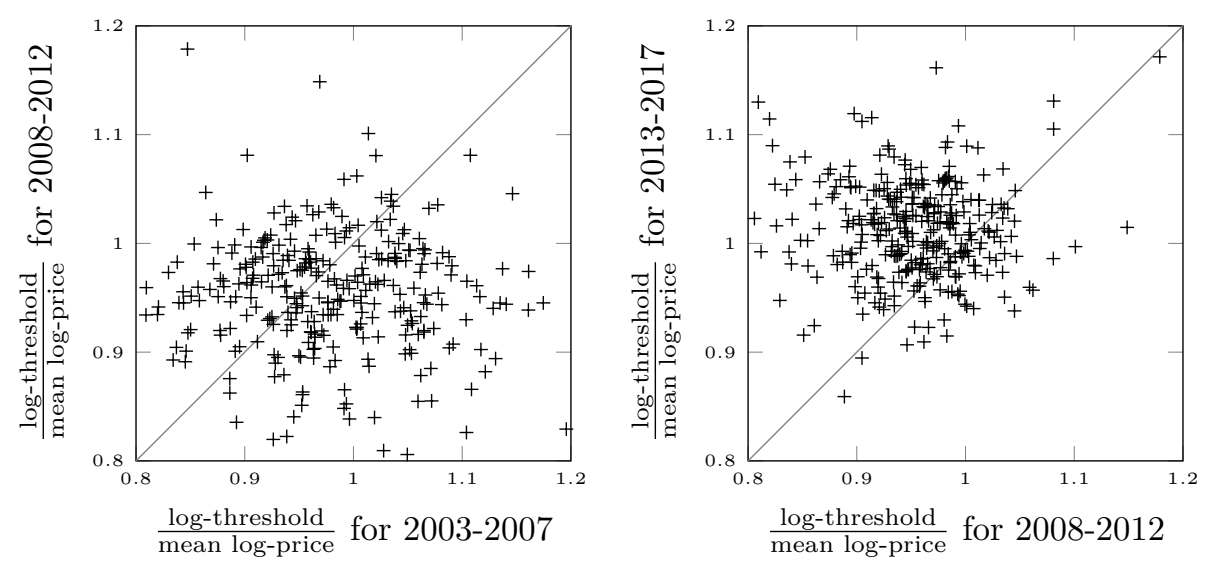

Figure 6: Ratio of the log-threshold $r$ over the mean log-price for two consecutive periods. The solid line is $y=x$.

These facts seem to indicate than in the crisis period, a stronger leverage effect is more likely to occur at a lower threshold.

\section{Conclusion}

Leverage effects in finance have been the subject of a large literature with many empirical evidences. The geometric oscillating Brownian motion (GOBM) studied in this article mimics such leverage effect. This model can be interpreted 
as a continuous-time version of the self-exciting threshold autoregressive model (SETAR).

We have shown its validity on real data and exhibited evidence in favor of leverage effects. We detect a mean-reverting behavior for most of the stocks in periods of financial crisis, less so in periods not containing major events, in agreement with Spierdijk, Bikker \& Hoek (2012). Our estimations are consistent with the ones of M. Esquível and P. Mota based on least squares.

Our model is simple and does not aim at capturing other stylized facts. It could serve as a basic building brick for more complex models. Our rationale is that the GOBM is really tractable while offering more flexibility than the Black-Scholes model:

- The estimation procedure is simple to set up.

- Simulations are easily performed.

- The market is complete.

- Option pricing could be performed through analytic or semi-analytic approach without relying on Monte Carlo simulations.

In addition, our model and estimation procedure could serve other purposes. In this model the leverage effect is a consequence of a spatial segmentation in which the dynamics of the price changes according to a threshold. The same estimation procedure could also be applied in short time windows in order to detect sharp changes, hence reflecting temporal changes, as for regime switching models involving Hidden Markov models.

Another possible application of the GOBM, and more generally of local volatilities with discontinuities, would be to introduce such features in more complex models. The properties we showed in the present paper and their capability of reproducing extreme skews in the implied volatility (Pigato 2017) suggest that such discontinuities could be a tractable way to introduce asymmetries and regime changes in other models (Decamps, Goovaerts \& Schoutens 2006).

\section{Acknowledgements}

P. Pigato gratefully acknowledges financial support from ERC via Grant CoG683164. The authors are grateful to the anonymous reviewers for their useful suggestions. 


\section{References}

Ait-Sahalia, Y., J. Fan \& Y. Li (2013). "The Leverage Effect Puzzle: Disentangling Sources of Bias at High Frequency". In: Journal of Financial Economics 109, pp. 224-249.

Akaike, H. (1973). "Information theory and an extension of the maximum likelihood principle". In: Second International Symposium on Information Theory (Tsahkadsor, 1971). Akadémiai Kiadó, Budapest, pp. 267-281.

Alvarez, E., H. R. Luis \& Paavo Salminen (2017). "Timing in the presence of directional predictability: optimal stopping of skew Brownian motion". In: Math. Methods Oper. Res. 86.2, pp. 377-400. DOI: 10.1007/s00186-017-0602-4.

Ang, A. \& A. Timmermann (2012). "Regime Changes and Financial Markets". In: Annual Review of Financial Economics 4, pp. 313-337. DOI: 10.1146/annurevfinancial-110311-101808.

Ankirchner, Stefan, Christophette Blanchet-Scalliet \& Monique Jeanblanc (2017). "Controlling the occupation time of an exponential martingale". In: Applied Mathematics and Optimization 76.2, pp. 415-428. DOI: $10.1007 / \mathrm{s} 00245-016-$ 9356-2.

Barndorff-Nielsen, Ole E. \& Neil Shephard (2002). "Econometric analysis of realized volatility and its use in estimating stochastic volatility models". In: J. R. Stat. Soc. Ser. B Stat. Methodol. 64.2, pp. 253-280. DoI: 10.1111/1467-9868.00336.

Black, F. (1976). "Studies of Stock Price Volatility Changes". In: Proceedings of the 1976 Meetings of the Business and Economical Statistics Section. American Statistical Association, pp. 177-181.

Brockwell, P. J.\&R. J. Williams (1997). "On the existence and application of continuous-time threshold autoregressions of order two". In: Adv. in Appl. Probab. 29.1, pp. 205-227. DOI: $10.2307 / 1427867$.

Chan, K. S. \& O. Stramer (1998). "Weak consistency of the Euler method for numerically solving stochastic differential equations with discontinuous coefficients". In: Stochastic Process. Appl. 76.1, pp. 33-44. DOI: 10.1016/S0304-4149(98)000209.

Chen, Cathy W. S., F. C. Liu \& Mike K. P. So (2008). "Heavy-tailed distributed threshold stochastic volatility models in financial time series". In: Australian 8 New Zealand Journal of Statistics 50.1, pp. 29-51.

Chen, Cathy W. S., Mike K. P. So \& Feng-Chi Liu (2011). "A review of threshold time series models in finance". In: Statistics and its Interface 4.2, pp. 167-181.

Chen, Cathy W.S. \& Mike K.P. So (2006). "On a threshold heteroscedastic model". In: International Journal of Forecasting 22.1, pp. 73-89. URL: https://ideas repec.org/a/eee/intfor/v22y2006i1p73-89.html. 
Christie, A. A. (1982). "The stochastic behavior of common stock variances: Value, leverage and interest rate effects". In: Journal of Financial Economics 10, pp. 407432. DOI: 10.1016/0304-405X (82) 90018-6.

Cont, R. (2001). "Empirical properties of asset returns: stylized facts and statistical issues". In: Quantitative Finance 1.2, pp. 223-236. DOI: 10.1080/713665670.

Decamps, Marc, Ann De Schepper \& Marc Goovaerts (2004). "Applications of $\delta$ function perturbation to the pricing of derivative securities". In: Phys. A 342.3-4, pp. 677-692. DOI: 10.1016/j.physa.2004.05.035.

Decamps, Marc, Marc Goovaerts \& Wim Schoutens (2006). "Self exciting threshold interest rates models". In: Int. J. Theor. Appl. Finance 9.7, pp. 1093-1122. DOI: 10.1142/S0219024906003937.

Engle, Robert F., Sergio M. Focardi \& Frank J. Fabozzi (2012). "ARCH/GARCH Models in Applied Financial Econometrics". In: Encyclopedia of Financial Models. John Wiley \& Sons, Inc. DOI: 10.1002/9781118182635. efm0062.

Esquível, Manuel L. \& Pedro P. Mota (2014). "On some auto-induced regime switching double-threshold glued diffusions". In: J. Stat. Theory Pract. 8.4, pp. 760-771. DOI: $10.1080 / 15598608.2013 .854184$.

Étoré, Pierre (2006). "On random walk simulation of one-dimensional diffusion processes with discontinuous coefficients". In: Electron. J. Probab. 11, no. 9, 249-275. DOI: $10.1214 /$ EJP.v11-311.

Gairat, Alexander \& Vadim Shcherbakov (2016). "Density of Skew Brownian motion and its functionals with application in finance". In: Mathematical Finance 26.4, 1069-1088. DOI: $10.1111 / \mathrm{mafi} .12120$.

Hens, T.\& S. C. Steude (2009). "The leverage effect without leverage: An experimental study". In: Finance Research Letter 6.2, pp. 83-94. DOI: $10.1016 / \mathrm{j} . \mathrm{frl}$ 2009.01.002.

Hottovy, S. \& S. N. Stechmann (2015). "Threshold models for rainfall and convection: Deterministic versus stochastic triggers". In: SIAM Journal on Applied Mathematics 75.2, pp. 861-884. DOI: 10.1137/140980788.

Iacus, Stefano M. (2008). Simulation and inference for stochastic differential equations, with $R$ examples. Springer Series in Statistics. Springer, New York. DOI: 10.1007/978-0-387-75839-8

Jang, Bong-Gyu et al. (2015). "Psychological Barriers and Option Pricing". In: Journal of Futures Markets 35.1, pp. 52-74. DOI: 10.1002/fut.21648.

Jeanblanc, Monique, Marc Yor \& Marc Chesney (2009). Mathematical methods for financial markets. Springer Finance. Springer-Verlag London, Ltd., London. DOI: 10.1007/978-1-84628-737-4

Keilson, Julian \& Jon A. Wellner (1978). "Oscillating Brownian motion". In: J. Appl. Probability 15.2, pp. 300-310. 
Kolb, Aaron (2016). Strategic real options. Working paper. Indiana University Kelley School of Business. URL: https://site.stanford.edu/sites/default/ files/strategicrealoptions.pdf.

Kutoyants, Yury A. (2004). Statistical inference for ergodic diffusion processes. Springer Series in Statistics. Springer-Verlag London, Ltd., London. DOI: 10. 1007/978-1-4471-3866-2.

Le Gall, J.-F. (1984). "One-dimensional stochastic differential equations involving the local times of the unknown process". In: Stochastic analysis and applications (Swansea, 1983). Vol. 1095. Lecture Notes in Math. Springer, Berlin, pp. 51-82. DOI: $10.1007 / \mathrm{BFb} 0099122$.

Lejay, A., L. Lenôtre \& G. Pichot (2017). Analytic expressions of the solutions of advection-diffusion problems in $1 D$ with discontinuous coefficients. Preprint.

Lejay, A.\& P. Pigato (2017). Data and methods for A threshold model for local volatility: evidence of leverage and mean reversion effects on historical data. Technical Report 0494. Inria.

Lejay, Antoine \& Paolo Pigato (2018a). Estimation of the drift parameters of a Drifted Oscillating Brownian motion. Preprint.

- (2018b). "Statistical estimation of the Oscillating Brownian Motion". In: Bernoulli 24.4B, pp. 3568-3602. DOI: 10.3150/17-BEJ969.

Lenôtre, Lionel (2015). "Study and Simulation of Skew Diffusion Processes". Ph.D. thesis. Université Rennes 1, France.

Lipton, Alex \& Artur Sepp (2011). "Filling the gaps". In: Risk Magazine, pp. 66-71.

Lipton, Alexander (2018). "Oscillating Bachelier and Black-Scholes Formulas". In: Financial Engineering. World Scientific.

Lo, Andrew W. \& A. Craig MacKinlay (1988). "Stock Market Prices Do Not Follow Random Walks: Evidence from a Simple Specification Test". In: The Review of Financial Studies 1.1, p. 41. DOI: 10.1093/rfs/1.1.41.

Meng, Hui et al. (2013). "Optimal portfolio in a continuous-time self-exciting threshold model". In: J. Ind. Manag. Optim. 9.2, pp. 487-504. DOI: 10.3934/ jimo.2013.9.487.

Monoyios, Michael \& Lucio Sarno (2002). "Mean reversion in stock index futures markets: A nonlinear analysis". In: Journal of Futures Markets 22.4, pp. 285-30.

Mota, Pedro P. \& Manuel L. Esquível (2014). "On a continuous time stock price model with regime switching, delay, and threshold". In: Quant. Finance 14.8, pp. 1479-1488. DOI: 10.1080/14697688.2013.879990.

Pai, J. \& H. Pedersen (1999). "Threshold Models of the Term Structure of Interest Rate". In: Joint day Proceedings Volume of the XXXth International ASTIN Colloquium/9th International AFIR Colloquium, Tokyo, Japan, pp. 387-400.

Pigato, P. (2017). Extreme at-the-money skew in a local volatility model. Preprint WIAS 2468. 
Poterba, James M.\& Lawrence H. Summers (1988). "Mean reversion in stock prices". In: Journal of Financial Economics 22.1, pp. 27-59. DOI: 10.1016/0304405X (88) 90021-9.

Priestley, M. B. (1988). Nonlinear and nonstationary time series analysis. Academic Press, Inc.

Rabemananjara, R.\& J. M. Zakoian (1993). "Threshold ARCH models and asymmetries in volatility". In: Journal of Applied Econometrics 8.1, pp. 31-49.

Rossello, Damiano (2012). "Arbitrage in skew Brownian motion models". In: Insurance Math. Econom. 50.1, pp. 50-56. DOI: 10.1016/j.insmatheco.2011.10. 004.

Salhi, Khaled et al. (2016). "Regime switching model for financial data: empirical risk analysis". In: Physica A 461, pp. 148-157. DOI: 10.1016/j .physa.2016.05.002

Siu, Tak Kuen (2016). "A self-exciting threshold jump-diffusion model for option valuation". In: Insurance Math. Econom. 69, pp. 168-193. DOI: $10.1016 / \mathrm{j}$ insmatheco.2016.05.008.

So, Mike K. P., W. K. Li \& K. Lam (2002). "A threshold stochastic volatility model". In: Journal of Forecasting 21.7, pp. 473-500.

Spierdijk, Laura, Jacob A. Bikker \& Pieter van den Hoek (2012). "Mean reversion in international stock markets: An empirical analysis of the 20th century". In: Journal of International Money and Finance 31.2, pp. 228-249. DOI: 10.1016/ j. jimonfin.2011.11.008.

Su, Fei \& Kung-Sik Chan (2015). "Quasi-likelihood estimation of a threshold diffusion process". In: J. Econometrics 189.2, pp. 473-484. DOI: 10.1016/j.jeconom. 2015.03 .038

- (2016). "Option pricing with threshold diffusion processes". In: N. Am. Actuar. J. 20.2, pp. 133-141. DOI: 10.1080/10920277.2015.1106953.

- (2017). "Testing for threshold diffusion". In: J. Bus. Econom. Statist. 35.2, pp. 218-227. DOI: $10.1080 / 07350015.2015 .1073594$

Tong, H. (2011). "Threshold models in time series analysis - 30 years on". In: Statistics and its Interface 4.

Tong, Howell (1983). Threshold models in nonlinear time series analysis. Vol. 21. Lecture Notes in Statistics. Springer-Verlag, New York. DoI: 10.1007/978-14684-7888-4.

- (2015). "Threshold models in time series analysis - some reflections". In: $J$. Econometrics 189.2, pp. 485-491. DOI: j.jeconom.2015.03.039.

$\mathrm{Xu}$, Dinghai (2012). "Examining realized volatility regimes under a threshold stochastic volatility model". In: Int. J. Fin. Econ. 17, pp. 373-389. DOI: 10 1002/ijfe.1458. 
Yadav, P.K., P.F. Pope \& K. Paudyal (1994). "Threshold autoregressive modeling in finance: The price differences of equivalent assets". In: Mathematical Finance 4.2, pp. 205-221. DOI: $10.1111 / \mathrm{j} .1467-9965.1994 . t b 00058 . x$.

Yan, Liqing (2002). "The Euler scheme with irregular coefficients". In: Ann. Probab. 30.3, pp. 1172-1194. DOI: 10.1214/aop/1029867124. 